\title{
Synthesis and Identification of Some New Tetrazole Derivatives from 4,5-dichloro Imidazole and Study of Their Biological Activity
}

Zainab fadhil and Shaimaa Adnan

Department of chemistry, College of Education, Univeristy of AI-Qadisiyah, Iraq

\begin{abstract}
A number of tetrazoles derivatives were prepared by series of steps, the first step includes react (4,5-dichloroimidazole) with (4-aminoacetophenone) to form 1-(4-((4,5-dichloro-1H-imidazol-2yl)diazenyl)ethanone (1). The second step was (1) reaction with (Amines derivatives) to get Schiff bases (2-7). The third step was reaction of Shciff base with sodiumazide to form tetrazoles derivatives (8-13). then study the biological activity for all compounds to word two type of bacteria.
\end{abstract}

Key words: Azo, Schiff base, tetrazole, imidazole, biological activity.

\section{Introduction}

Azo compounds are very well known and constitute one of the most varied groups of synthetic organic dyes, which are used in the textile, coloring agents for foods paper, cosmetics industries and technologies like liquid crystals $[1,2]$.

Azo imine compounds are important in applications different fields of science chemically and biologically. They are used in dyeing textiles, non-linear optical properties, optical switching and preparation of photoactive materials [3-5].

Tetrazole are aromatic five membered ring containing four nitrogen atoms. The first tetrazole was reported over a century ago [6-8].

Tetrazole and its derivatives have attracted much attention because of their applications as antimicrobial agents, anticancer, antifungal, a series of triazole containing tetrazole evaluated for their activity as antinociceptive and anti-inflammatory agents, anticonvulsant activity, antidiabetic activity, antihypertensive activity.

Tetrazole derivatives have considerable importance

Corresponding author: Dr. Shaimaa Adnan Behget, assisant professor, research field: organic chemistry.
[9], its applications as antimicrobial agents, antifungal, anticancer its activity as antinociceptive [10] and anti-inflammatory [11] agents, antidiabetic activity [12], anticonvulsant activity [13, 14], antihypertensive activity [15].

\section{Materials}

(FTIR) Spectra $\left(400-4000 \mathrm{~cm}^{-1}\right)$ in $\mathrm{KBr}$ disk were recorded on a SHIMADZU FTIR-8400S Fourier transform. Melting points were measured using Stuart, UK. ${ }^{13} \mathrm{C}-\mathrm{NMR}$ and ${ }^{1} \mathrm{HNMR}$ were recorded on Fourier transformation Bruker spectrometer operating at $(400 \mathrm{MHz})$ with (DMSO-ds) measurements were made at Department of Chemistry, Kashan University, Iran.

\subsection{Synthesis Azo Derivative}

1-(4-((4,5-dichloro-1H-imidazol-2-yl) diazenyl) phenyl) ethanone (1) (4-aminoacetophenone) (0.005 mmol, $0.0675 \mathrm{mgm}$ ) of the aromatic amine was dissolved in $5 \mathrm{~mL}$ of concentrated $\mathrm{HCl}$ and $7 \mathrm{~mL}$ of distilled water. The mixture is cooled to $0{ }^{\circ} \mathrm{C}$ and (0.005 mmol, 0.345$)$ of sodiumnitrite to add dropwise with continuous stirring. The solution was left for 15 mins to stable after completing the addition $(0.005$ 
$\mathrm{mmol})$ of (4,5-dichloroimidazole) dissolved in $(1 \mathrm{~mL}$ $\mathrm{NaOH}$ IN $40 \mathrm{~mL} \quad \mathrm{H}_{2} \mathrm{O}$ ) was added, a orange precipitate was formed, filtered and recrystallization from ethanol [16].

\subsection{General Method of Synthesis Schiff Base $(2,3,4,5,6,7)$}

A mixture of equimolar quantities $(0.01 \mathrm{~mol})$ of (1) and (4-chloroaniline, 4-bromoaniline, 5-nitro-2-aminothiazol, 3-aminoacetophenone, 2-aminobenzimidazole, 4,4-methylenedianiline) (0.01 mol) were refluxed for 2-3 $\mathrm{h}$ in $5 \mathrm{~mL}$ of ethanol alcohol, then it was added (2) drops of glacial acetic acid as catalyst. The mixture was cooled and kept for $24 \mathrm{~h}$, the crystals found were filtered, dried and recrystallization from ethanol alcohol to give $(2,3,4,5,6)$ consecutive [17].

\subsection{Synthesis of Tetrazole Derivative $(8,9,10,11,13,13)$}

A mixture of Schiff base $(2,3,4,5,6,7)(0.001$ $\mathrm{mol})$ dissolved in 1,4-dioxane $(15 \mathrm{~mL})$ and sodium azide $(0.001 \mathrm{~mol})$ was dissolved in 1,4-dioxan $(15 \mathrm{~mL})$ and refluxed for 14-24 h. The reaction was cooled and the resulting final $(8,9,10,11,12,13)$ consecutive recrystallization from ethanol [18].

\subsection{Preparation of Microbiology Culture Media}

$20 \mathrm{~g}$ of nutrient agar is dissolved in $(500 \mathrm{~mL})$ of distillation water, then put in autoclave for $20 \mathrm{mins}$ at $200{ }^{\circ} \mathrm{C}$ for sterilization. Pouring the media after becoming at $37{ }^{\circ} \mathrm{C}$ in Petri dishes, made ready for streaking by bacteria. It was getting (Escherichia coli) and (staphylococcus aurous) isolated bacteria from hospital. It was cultured and these plates were incubated at $37^{\circ} \mathrm{C}$ for $24 \mathrm{~h}$ for both bacteria [19].

\section{Results and Discussion}

3.1 Compound (1): 1-(4-((4,5-dichloro-1H-imidazol2-yl)diazenyl)phenyl)ethanone

The infrared spectrum data of compound (1) showed band at $(1,720) \mathrm{cm}^{-1}$ for $(\mathrm{C}=\mathrm{O}), 3,039 \mathrm{~cm}^{-1}$ for (Ar-H), 3,201 $\mathrm{cm}^{-1}$ for(N-H) imidazole, $1,666 \mathrm{~cm}^{-1}$ for $(\mathrm{C}=\mathrm{N})$ inside imidazole ring, $1,965 \mathrm{~cm}^{-1}$ for $(\mathrm{C}-\mathrm{H})$ for $\left(\mathrm{CH}_{3}\right), 1,480 \mathrm{~cm}^{-1}$ for $(\mathrm{N}=\mathrm{N})$ and $880 \mathrm{~cm}^{-1}$ for $(\mathrm{C}-\mathrm{Cl})$.

The ${ }^{1} \mathrm{H}$ NMR (DMSO) spectrum data of compound (1) show $\delta$ : 6.9-8.1 (m.,4H, Ar-H), $4.2\left(\mathrm{~S}, 3 \mathrm{H}, \mathrm{CH}_{3}\right)$, 11.2 (S, 1H, N-H imidazole ring).

The ${ }^{13} \mathrm{C}-\mathrm{NMR}$ (DMSO) spectrum data of compound (1) show $\delta$ : $149\left(\mathrm{C} 3, \mathrm{C}_{2}\right), 197\left(\mathrm{C}_{10}\right), 157\left(\mathrm{C}_{1}\right), 26\left(\mathrm{C}_{11}\right)$, $135\left(\mathrm{C}_{7}\right), 133\left(\mathrm{C}_{4}\right), 130-110 \mathrm{C}$ arom.

3.2 Compound (2): 4-chloro- $N-(1-(4-((4,5-d i c h l o r o-$ 1H-imidazol-2-yl)diazenyl)phenyl)ethylidene) aniline

The infrared spectrum data of compound (2) showed band at $3,055 \mathrm{~cm}^{-1}$ for $(\mathrm{Ar}-\mathrm{H}), 3,116 \mathrm{~cm}^{-1}$ for (N-H) imidazole, $1,610 \mathrm{~cm}^{-1}$ for $(\mathrm{C}=\mathrm{N}), 1,458 \mathrm{~cm}^{-1}$ for $(\mathrm{N}=\mathrm{N}), 650 \mathrm{~cm}^{-1}$ for $(\mathrm{C}-\mathrm{Br})$.

\subsection{Compound (3): 4-bromo-N-(1-(4-((4,5-dichloro-} 1H-imidazol-2yl)diazenyl)phenyl)ethylidene)aniline

The infrared spectrum data of compound (3) showed band at $3,055 \mathrm{~cm}^{-1}$ for (Ar-H), 3,116 $\mathrm{cm}^{-1}$ for (N-H) imidazole, $1,610 \mathrm{~cm}^{-1}$ for $(\mathrm{C}=\mathrm{N}), 1,458 \mathrm{~cm}^{-1}$ for $(\mathrm{N}=\mathrm{N}) 650 \mathrm{~cm}^{-1}$ for $(\mathrm{C}-\mathrm{Br})$. The ${ }^{1} \mathrm{H}$ NMR (DMSO) spectrum data of compound (3) show $\delta: 7.1-7.8$ (m., 8, Ar-H), 10.7 (S, 2H, NH imidazol ring), 1.9 (S, 3H, $\mathrm{CH}_{3}$ ).

The ${ }^{13} \mathrm{C}-\mathrm{NMR}$ (DMSO) spectrum data of compound (3) show $\delta$ : $141\left(\mathrm{C}_{3}, \mathrm{C}_{2}\right), 163\left(\mathrm{C}_{10}\right), 157\left(\mathrm{C}_{12}\right), 149$ $\left(\mathrm{C}_{1}\right), 61\left(\mathrm{C}_{11}\right), 1,268\left(\mathrm{C}_{13}\right), 132\left(\mathrm{C}_{7}\right), 130\left(\mathrm{C}_{4}\right)$, 120-142 Carom.

3.4 Compound (4): N-(1-(4-((4,5-dichloro-1H-imida zol-2-yl)diazenyl)phenyl)ethylidene)-5-nitrothiazol-2amine

The infrared spectrum data of compound (4) showed band at $1,250 \mathrm{~cm}^{-1}$ for (C-S), $3,016 \mathrm{~cm}^{-1}$ for (Ar-H), 2,180 $\mathrm{cm}^{-1}$ for $(\mathrm{N}-\mathrm{H})$ imidazole, $1,596 \mathrm{~cm}^{-1}$ for $(\mathrm{C}=\mathrm{N})$ inside imidazole ring, $836 \mathrm{~cm}^{-1}$ for $(\mathrm{C}-\mathrm{Cl})$ $1,566 \mathrm{~cm}^{-1}$ for $(\mathrm{C}=\mathrm{C})$ Aromatic. 


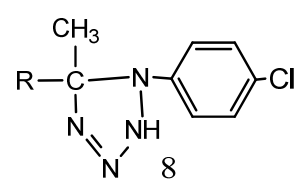<smiles>[R]C1(C)N=NNN1c1ccc(Br)cc1</smiles><smiles>[R]C1(C)N=NNN1c1ncc([N+](=O)[O-])s1</smiles>

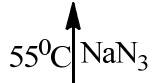<smiles>c1ccncc1</smiles>

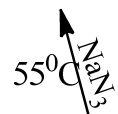<smiles>[R]C(C)=Nc1ccc(Br)cc1</smiles>

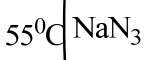<smiles>[R]Nc1ccc(Cl)cc1</smiles>

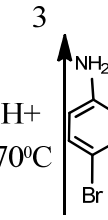<smiles></smiles><smiles>O=C(Cl)c1ccc(N=Nc2nc(Cl)c(Cl)[nH]2)cc1</smiles>

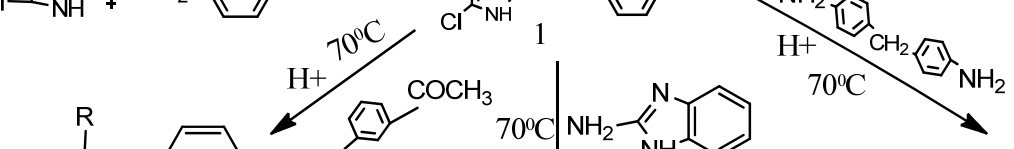<smiles>[R]CN=C(C)c1ccc(Cc2ccc(N=C([R])C)cc2)cc1</smiles>

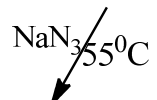<smiles>[R]C1(C)N=NNN1c1cccc(C(C)=O)c1</smiles>

11<smiles>[R]C(C)=Nc1nc2ccccc2[nH]1</smiles>

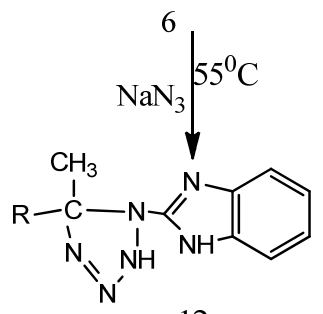<smiles></smiles>

Fig. 1 Scheme of preparation Tetrazole derivatives.

3.5 Compound (5): 1-(3-((1-(4-)((4,5-dichloro-1H-im idazol-2-yl)diazenyl)phenyl)ethylidene)amino)phenyl) ethanone

The infrared spectrum data of compound (5) showed band at $1,740 \mathrm{~cm}^{-1}$ for $(\mathrm{C}=\mathrm{O}), 3,040 \mathrm{~cm}^{-1}$ for (Ar-H), 3,220 $\mathrm{cm}^{-1}$ for (N-H) imidazole, $1,580 \mathrm{~cm}^{-1}$ for $(\mathrm{C}=\mathrm{N})$ inside imidazole ring, $2,955 \mathrm{~cm}^{-1}$ for $(\mathrm{H}-\mathrm{H})$ in $\mathrm{CH}_{3} 1,566 \mathrm{~cm}^{-1}$ for $(\mathrm{C}=\mathrm{C})$ Aromatic.

3.6 Compound (6): $\mathrm{N}$-(1-(4-((4,5-dichloro-1H-imida zol-2-yl)diazenyl) phenyl) ethylidene)-1H-benzo [d] imidazol-2-amine

The infrared spectrum data of compound (6) showed band at $3,020 \mathrm{~cm}^{-1}$ for $(\mathrm{Ar}-\mathrm{H}), 3,160 \mathrm{~cm}^{-1}$ for $(\mathrm{N}-\mathrm{H})$ imidazole, $1,566 \mathrm{~cm}^{-1}$ for $(\mathrm{C}=\mathrm{N})$ inside imidazole ring, 1,546$) \mathrm{cm}^{-1}$ for $(\mathrm{C}=\mathrm{C})$ Aromatic.

3.7 Compound (7): 4,4'-methylenebis(N-(1-(4-((4,5dichloro-1H-imidazol-2-yl)diazenyl)phenyl)ethylidene) aniline)

The infrared spectrum data of compound (7) showed band at $2,931 \mathrm{~cm}^{-1}$ for $(\mathrm{C}-\mathrm{H})$ in $\left(\mathrm{CH}_{3}\right)$, $3,024 \mathrm{~cm}^{-1}$ for $(\mathrm{Ar}-\mathrm{H}), \quad 3,132 \mathrm{~cm}^{-1}$ for $(\mathrm{N}-\mathrm{H})$ imidazole, $1,598 \mathrm{~cm}^{-1}$ for $(\mathrm{C}=\mathrm{N})$ inside Imidazole ring, $840 \mathrm{~cm}^{-1}$ for $(\mathrm{C}-\mathrm{Cl}), 1,566 \mathrm{~cm}^{-1}$ for $(\mathrm{C}=\mathrm{C})$ Aromatic. 
3.8 Compound (8): 2-(1H-benzo [d] imidazol-2-yl) -4(5-(4-(dimethylamino)phenyl)-1H-tetrazol-1-yl)phenol

The infrared spectrum data of compound (8) showed band at $3,178 \mathrm{~cm}^{-1}$ for $(\mathrm{N}-\mathrm{H})$ in tetrazole, $3,055 \mathrm{~cm}^{-1}$ for $(\mathrm{Ar}-\mathrm{H}), 2,977 \mathrm{~cm}^{-1}$ for $(\mathrm{C}-\mathrm{H})$ in $\mathrm{CH}_{3}$, $1,566 \mathrm{~cm}^{-1}$ for $(\mathrm{C}=\mathrm{C})$ Aromatic.

The ${ }^{1} \mathrm{H}$ NMR (DMSO) spectrum data of compound (8) show $\delta$ : 6.5-7.6 (m., 8H, Ar-H), $10.3(\mathrm{~S}, 1 \mathrm{H}, \mathrm{H}-\mathrm{N}$ Tetrazole) $10.1(\mathrm{~S}, 1 \mathrm{H}, \mathrm{N}-\mathrm{H})$ imidazole.

The ${ }^{13} \mathrm{C}-\mathrm{NMR}$ (DMSO) spectrum data of compound (8) show $\delta: 125\left(\mathrm{C}_{3}, \mathrm{C}_{2}\right), 137\left(\mathrm{C}_{12}\right), 128\left(\mathrm{C}_{15}\right), 130$ $\left(\mathrm{C}_{1}\right), 66\left(\mathrm{C}_{11}\right), 129\left(\mathrm{C}_{7}\right), 126\left(\mathrm{C}_{3}, \mathrm{C}_{4}\right)$.

3.9 Compound (9): 1-(4-bromophenyl)-5-(4-((4,5-di chloro-1H-imidazol-2-yl)diazenyl)phenyl)-5-methyl-2, 5-dihydro-1H-tetrazole

The infrared spectrum data of compound (9) showed band at $3,180 \mathrm{~cm}^{-1}$ for $(\mathrm{N}-\mathrm{H})$ intetrazole, $3,016 \mathrm{~cm}^{-1}$ for $(\mathrm{Ar}-\mathrm{H}), 2,977 \mathrm{~cm}^{-1}$ for $(\mathrm{C}-\mathrm{H})$ in $\mathrm{CH}_{3}$, $1,419 \mathrm{~cm}^{-1}$ for $(\mathrm{N}=\mathrm{N}), 1,566 \mathrm{~cm}^{-1}$ for $(\mathrm{C}=\mathrm{C})$ Aromatic.

The ${ }^{1} \mathrm{H}$ NMR (DMSO) spectrum data of compound (9) show $\delta$ : 6.5-7.8 (m., 8H, Ar-H), $9.6(\mathrm{~S}, 1 \mathrm{H}, \mathrm{NH}$ Amidazol ring), 10.5 (S, 1HN-H tetrazole, 3.8 (S, 3H, $\left.\mathrm{CH}_{3}\right)$.

The ${ }^{13} \mathrm{C}$-NMR (DMSO) spectrum data of compound (9) show $\delta$ : $158\left(\mathrm{C}_{10}\right), 155\left(\mathrm{C}_{12}\right), 160\left(\mathrm{C}_{1}\right), 62\left(\mathrm{C}_{11}\right)$, $137\left(\mathrm{C}_{1}\right), 123\left(\mathrm{C}_{6}, \mathrm{C}_{7}\right), 148\left(\mathrm{C}_{4}\right)$ 133-112 (Carom).

3.10 Compound (10): 2-(5-(4-((4,5-dichloro-1H-imi dazol-2-yl)diazenyl)phenyl)-5-methyl-2,5-dihydro- $1 \mathrm{H}$ tetrazol-1-yl)-5-nitrothiazole

The infrared spectrum data of compound (10) showed band at $3,223 \mathrm{~cm}^{-1}$ for $(\mathrm{N}-\mathrm{H})$ in tetrazole, $3,055 \mathrm{~cm}^{-1}$ for $(\mathrm{Ar}-\mathrm{H}), 1,450 \mathrm{~cm}^{-1}$ for $(\mathrm{N}=\mathrm{N})$ inside imidazole ring, $840 \mathrm{~cm}^{-1}$ for (C-Cl).

The ${ }^{1} \mathrm{H}$ NMR (DMSO) spectrum data of compound (10) show $\delta$ : 7-8.3 (m., 5H, Ar- $\mathrm{H}), 1.2\left(\mathrm{~S}, 3 \mathrm{H}, \mathrm{CH}_{3}\right)$, $9.1(\mathrm{~S}, 1 \mathrm{H}, \mathrm{NH}$ Amidazol ring), $9.3(1 \mathrm{H}, \mathrm{NH}$, tetrazole).

The ${ }^{13} \mathrm{C}$-NMR (DMSO) spectrum data of compound (10) show $\delta$ : $147\left(\mathrm{C}_{12}\right), 125.2\left(\mathrm{C}_{1}\right), 128.7$
$\left(\mathrm{C}_{11}\right), 128.7\left(\mathrm{C}_{13}\right) 128.14\left(\mathrm{C}_{14}\right), 112-125$ (Carom).

3.11 Compound (11): 1-(3-(5-(4-((4,5-dichloro- $1 \mathrm{H}-\mathrm{i}$ midazol-2-yl)diazenyl)phenyl)-5-methyl-2,5-dihydro1H-tetrazol-1-yl)phenyl)ethanone

The infrared spectrum data of compound (11) showed band at $3,224 \mathrm{~cm}^{-1}$ for $(\mathrm{N}-\mathrm{H})$ in tetrazole, $3,016 \mathrm{~cm}^{-1}$ for (Ar-H), $3,163 \mathrm{~cm}^{-1}$ for $(\mathrm{N}-\mathrm{H})$ imidazole, $1,419 \mathrm{~cm}^{-1}$ for $(\mathrm{N}=\mathrm{N}), 636 \mathrm{~cm}^{-1}$ for $(\mathrm{C}-\mathrm{Br})$.

The ${ }^{1} \mathrm{H}$ NMR (DMSO) spectrum data of compound (11) show $\delta$ : 6.2-8 (m., 8H, Ar- H), 10.1 (S, $1 \mathrm{H}, \mathrm{N}-\mathrm{H}$ tetrazole), $9.8(\mathrm{~S}, 1 \mathrm{H}, \mathrm{NH}$ Amidazol ring), $3.2(\mathrm{~S}, 3 \mathrm{H}$, $\left.\mathrm{OCH}_{3}\right), 1.3\left(\mathrm{~S}, 3 \mathrm{H}, \mathrm{CH}_{3}\right)$.

The ${ }^{13} \mathrm{C}-\mathrm{NMR}$ (DMSO) spectrum data of compound (11) show $\delta$ : $113\left(\mathrm{C}_{5}, \mathrm{C}_{2}\right), 137\left(\mathrm{C}_{10}\right), 157$ $\left(\mathrm{C}_{12}\right), 190\left(\mathrm{C}_{18}\right), 138\left(\mathrm{C}_{1}\right), 61\left(\mathrm{C}_{11}\right), 62\left(\mathrm{C}_{19}\right), 141\left(\mathrm{C}_{7}\right)$.

3.12 Compound (12): 42-(5-(4-((4,5-dichloro- $1 \mathrm{H}-$ imidazol-2-yl)diazenyl) phenyl)-5-methyl-2,5-dihydro1H-tetrazol-1-yl)-1H-benzo[d] imidazole

The infrared spectrum data of compound (12) showed band at $3,130 \mathrm{~cm}^{-1}$ for $(\mathrm{N}-\mathrm{H})$ in tetrazole, $3,016 \mathrm{~cm}^{-1}$ for $(\mathrm{Ar}-\mathrm{H}), 1,450 \mathrm{~cm}^{-1}$ for $(\mathrm{N}=\mathrm{N}),(1,350$, $1,500) \mathrm{cm}^{-1}$ for $\left(\mathrm{NO}_{2}\right), 1.2\left(\mathrm{~s}, 3 \mathrm{H}, \mathrm{CH}_{3}\right)$.

The ${ }^{1} \mathrm{H}$ NMR (DMSO) spectrum data of compound (12) show $\delta$ : 6-7.8 (m., 6H, Ar-H), $10.4 \mathrm{~S}, 1 \mathrm{H} \mathrm{OH}$, $5.4\left(\mathrm{~S}, 2 \mathrm{H}, \mathrm{NH}_{2}\right), 10.6$ (S, 2H, NH Amidazol ring).

The ${ }^{13} \mathrm{C}-\mathrm{NMR}$ (DMSO) spectrum data of compound (12) show $\delta: 158\left(\mathrm{C}_{12}\right), 128\left(\mathrm{C}_{8}\right), 160\left(\mathrm{C}_{1}\right)$, $66\left(\mathrm{C}_{11}\right), 130\left(\mathrm{C}_{1}\right), 129\left(\mathrm{C}_{7}\right), 129\left(\mathrm{C}_{18}, \mathrm{C}_{17}\right), 111-125$ (Carom).

3.13 Compound (13): bis(4-(5-(4-((4,5-dichloro- $1 H-$ imidazol-2-yl)diazenyl)phenyl)-5-methyl-2,5-dihydro1H-tetrazol-1-yl)phenyl)methane

The infrared spectrum data of compound (13) showed band at $3,200 \mathrm{~cm}^{-1}$ for $(\mathrm{N}-\mathrm{H})$ in tetrazole, $3,055 \mathrm{~cm}^{-1}$ for $(\mathrm{Ar}-\mathrm{H}), 1,411 \mathrm{~cm}^{-1}$ for $(\mathrm{N}=\mathrm{N}), 2,923$ $\mathrm{cm}^{-1}$ for $(\mathrm{C}-\mathrm{H})$ in $\mathrm{CH}_{3}$.

The ${ }^{1} \mathrm{H}$ NMR (DMSO) spectrum data of compound (13) show $\delta$ : 6.2-8 (m, 16H, Ar- H), 10.1 (S, $1 \mathrm{H}, \mathrm{N}-\mathrm{H}$, tetrazole), $3.4\left(\mathrm{~S}, 2 \mathrm{H}, \mathrm{CH}_{2}\right), 9.2(\mathrm{~S}, 1 \mathrm{H}, \mathrm{NH}$ Amidazol 
ring), $1.2\left(\mathrm{~S}, 6 \mathrm{H}, \mathrm{CH}_{3}\right)$.

The ${ }^{13} \mathrm{C}-\mathrm{NMR}$ (DMSO) spectrum data of compound (13) show $\delta$ : $145\left(\mathrm{C}_{25}, \mathrm{C}_{10}\right), 61\left(\mathrm{C}_{26}, \mathrm{C}_{11}\right)$, $66\left(\mathrm{C}_{18}\right), 163\left(\mathrm{C}_{12}, \mathrm{C}_{22}\right), 159\left(\mathrm{C}_{1}, \mathrm{C}_{33}\right), 111-141$ (Carom).

\section{Conclusions}

In this work, the antibacterial test was performed according to the wells method. Compounds (1-30) were assayed for their antimicrobial activity in vitro against Gram-negative bacteria (Escherichia coli) and Gram-positive bacteria (staphylococcus aurous).
Prepared agar and Petri dishes were sterilized by autoclaving for $20 \mathrm{~min}$ at $200{ }^{\circ} \mathrm{C}$. These plates were incubated at $37{ }^{\circ} \mathrm{C}$ for $24 \mathrm{~h}$ for both bacteria. DMSO was used as a solvent to prepare solutions of the various compounds were examined ( $0.02 \mathrm{~g}$ of comp./5 $\mathrm{mL}$ DMSO). The inhibition zones caused by the various compounds were examined, where compounds which appeared good activity $(1,8,18$ and 28) against (staphylococcus aurous) on other hand; compounds (1 and 13) which appeared good activity against (Escherichia coli). The results of the preliminary screening tests are listed in Table 1 [25].

Table 1 Biological activity for compounds (1-13).

\begin{tabular}{llllll}
\hline Compounds No. & E. coli & Staph. aureus & Compounds No. & E. coli & Staph. aureus \\
\hline 1 & + & + & 8 & $\mathrm{R}$ & +++ \\
2 & + & +++ & 9 & $\mathrm{R}$ & +++ \\
3 & - & ++ & 10 & $\mathrm{R}$ & - \\
4 & - & ++ & 11 & $\mathrm{R}$ & ++ \\
5 & - & 12 & $\mathrm{R}$ & +++ \\
6 & - & 13 & + & ++ \\
7 & - & & & \\
$-=$ No inhibition $=$ inactive,$+=(5-10) \mathrm{mm}=$ slightly active, Resistance $(+-)=\mathrm{R}$ & & \\
$++=(11-20) \mathrm{mm}=$ moderately active,$+++=($ more than 20$) \mathrm{mm}=$ Good active.
\end{tabular}

Table 2 Physical properties of compounds (1-13).

\begin{tabular}{|c|c|c|c|c|c|c|c|}
\hline No. & Name of comp. & M. F & M. W & M.P $\left({ }^{\circ} \mathrm{C}\right)$ & $\mathrm{R}_{\mathrm{f}}$ & Colour & $\%$ \\
\hline 1 & 1-(4-((4,5-dichloro-1H-imidazol-2-yl) diazenyl) phenyl)ethanone & $\mathrm{C}_{11} \mathrm{H}_{8} \mathrm{Cl}_{2} \mathrm{~N}_{4} \mathrm{O}$ & 283 & $218-220$ & 0.3 & Orange & 83 \\
\hline 2 & $\begin{array}{l}\text { 4-chloro-N-(1-(4-((4,5-dichloro-1H-imidazol-2-yl) } \\
\text { diazenyl)phenyl)ethylidene)aniline }\end{array}$ & $\mathrm{C}_{17} \mathrm{H}_{12} \mathrm{Cl}_{3} \mathrm{~N}_{5}$ & 392 & $160-158$ & 0.3 & Black & 76 \\
\hline 3 & $\begin{array}{l}\text { 4-bromo-N-(1-(4-((4,5-dichloro-1H-imidazol-2-yl) } \\
\text { diazenyl)phenyl)ethylidene)aniline }\end{array}$ & $\mathrm{C}_{17} \mathrm{H}_{12} \mathrm{BrCl}_{2} \mathrm{~N}_{5}$ & 437 & $150-148$ & 0.2 & Black & 88 \\
\hline 4 & $\begin{array}{l}\mathrm{N}-(1-(4-((4,5 \text {-dichloro-1H-imidazol-2-yl) } \\
\text { diazenyl)phenyl)ethylidene)-5-nitrothiazol-2-amine }\end{array}$ & $\mathrm{C}_{14} \mathrm{H}_{9} \mathrm{Cl}_{2} \mathrm{~N}_{7} \mathrm{O}_{2} \mathrm{~S}$ & 410 & $186-184$ & 0.2 & Brown & 80 \\
\hline 5 & $\begin{array}{l}\text { 1-(3-((1-(4-((4,5-dichloro-1H-imidazol-2-yl) } \\
\text { diazenyl)phenyl)ethylidene)amino)phenyl)ethanone }\end{array}$ & $\mathrm{C}_{19} \mathrm{H}_{15} \mathrm{Cl}_{2} \mathrm{~N}_{5} \mathrm{O}$ & 400 & $145-143$ & 0.4 & Violt & 71 \\
\hline 6 & $\begin{array}{l}\text { N-(1-(4-((4,5-dichloro-1H-imidazol-2-yl)diazenyl)phenyl)ethylidene) } \\
\text {-1H-benzo[d]imidazol-2-amine }\end{array}$ & $\mathrm{C}_{18} \mathrm{H}_{13} \mathrm{Cl}_{2} \mathrm{~N}_{7}$ & 398 & $175-173$ & 0.3 & Red & 78 \\
\hline 7 & $\begin{array}{l}\text { lenebis(N-(1-(4-((4,5-dichloro-1H-imidazol-2-yl) } \\
\text { henyl)ethylidene)aniline) }\end{array}$ & $\mathrm{C}_{35} \mathrm{H}_{26} \mathrm{Cl}_{4} \mathrm{~N}_{10}$ & 728 & $174-175$ & 0.4 & Black & 71 \\
\hline 8 & $\begin{array}{l}\text { chloro-1H-imidazol-2-yl) } \\
\text { hydro-1H-tetrazole }\end{array}$ & $\mathrm{C}_{17} \mathrm{H}_{13} \mathrm{Cl}_{3} \mathrm{~N}_{8}$ & 434 & $150-148$ & 0.4 & Brown & 77 \\
\hline 9 & $\begin{array}{l}\text { 1-(4-bromophenyl)-5-(4-((4,5-dichloro-imidazol-2-yl) } \\
\text { diazenyl)phenyl)-5-methyl-2,5-dihydro-1H-tetrazole }\end{array}$ & $\mathrm{C}_{17} \mathrm{H}_{13} \mathrm{BrCl}_{2} \mathrm{~N}_{8}$ & 480 & $180-178$ & 0.3 & Brown & 76 \\
\hline 10 & $\begin{array}{l}\text { 2-(5-(4-((4,5-dichloro-imidazol-2-yl)diazenyl)phenyl) } \\
\text {-5-methyl-2,5-dihydro-1H-tetrazol-1-yl)-5-nitrothiazole }\end{array}$ & $\mathrm{C}_{14} \mathrm{H}_{10} \mathrm{Cl}_{2} \mathrm{~N}_{10} \mathrm{O}_{2} \mathrm{~S}$ & 453 & $300-298$ & 0.4 & Brown & 80 \\
\hline 11 & $\begin{array}{l}\text { 1-(3-(5-(4-((4,5-dichloro-imidazol-2-yl) diazenyl) phenyl) } \\
\text {-5-methyl-2,5-dihydro-1H-tetrazol-1-yl)phenyl) ethanone }\end{array}$ & $\mathrm{C}_{19} \mathrm{H}_{16} \mathrm{Cl}_{2} \mathrm{~N}_{8} \mathrm{O}$ & 443 & $150-148$ & 0.5 & Brown & 68 \\
\hline 12 & $\begin{array}{l}\text { 2-(5-(4-((4,5-dichloro-imidazol-2-yl)diazenyl)phenyl) } \\
\text {-5-methyl-2,5-dihydro-1H-tetrazol-1-yl)-1H-benzo[d]imidazole }\end{array}$ & $\mathrm{C}_{18} \mathrm{H}_{14} \mathrm{Cl}_{2} \mathrm{~N}_{10}$ & 441 & $157-155$ & 0.4 & Black & 72 \\
\hline 13 & $\begin{array}{l}\text { bis(4-(5-(4-((4,5--1H-imidazol-2-yl)diazenyl)phenyl) } \\
\text {-5-methyl-2,5-dihydro-1H-tetrazol-1-yl)phenyl)methane }\end{array}$ & $\mathrm{C}_{35} \mathrm{H}_{30} \mathrm{Cl}_{2} \mathrm{~N}_{16}$ & 814 & $165-163$ & 0.4 & Red & 71 \\
\hline
\end{tabular}




\section{References}

[1] Forgaty, W. M., and Kelly, C. T. 1979. Topics in Enzyme and Fermentation Biotechnology. Chichester: J. Wiley \& Sons.

[2] Noor, K. Z., and Wissam, M. M., 2016. "Storage of Serum in Plastic and Glass Containers May Alter the Serum Concentration of Polychlorinated Biphenyls." Baghdad Science Journal 13 (2): 868-75.

[3] Selma, B. A. L. 2016. "Novel Azo-Schiff Base Ligand and Its Cobalt, Copper, Nickel Complexes: Synthesis, Characterization, Antimicrobial, Catalytic and Electro Chemical Features." Appl. Sci. and Eng. 17 (2): 315-26.

[4] Belter, P. A., Cussler, E. L., and Hu, W. S. 1988. Bioseparations: Downstream Processing for Biotechnology. Minneapolis: John Wiley \& Sons.

[5] Ahamed, W. N., Hassan, T. G., and Abid, A. M. A., 2016. "Synthesis, Characterization and Antimicrobial Activity of Schiff Base (E)-N-(4- (2-Hydroxy Benzylideneamino) Phenylsulfonyl) Acetamide Metal Complexes." American Journal of Analytical Chemistry 7: 233-45.

[6] Greenberg, J. 2008. Of Prairie, Woods, and Water: Two Centuries of Chicago Nature Writing. Chicago: University of Chicago Press.

[7] Silverstein, T. 1974. Sir Gawain and the Green Knight. Chicago: University of Chicago Press.

[8] Sameaa, J. K., Selvana, A. Y., Amal, S. S., Tamador, A. M., and Zaynab, H. F. A. 2016. "Synthesis, Characterization of Derivatives Tetrazoles for Trimethoprim Drug." Baghdad Science Journal 13 (2): 266-74.

[9] Garcia, M. G. 1988. Love in the Time of Cholera. Translated by Grossman, E. London: Cape.

[10] Reed, B. W., Semmens, M. J., and Cussler, E. L. 1995. "Membrane Contactors." In Membrane Separation Technology, Principles and Application, edited by Noble, R. D., and Stern, S. A. Amesterdam: Elsevier.

[11] Faraday, C. Forthcoming. "Protean Photography." In Seven Trips beyond the Asteroid Belt, edited by Oring, J. Cape Canaveral, FL: Launch Press. AOAC (Association of Official Analytical Chemists). 2005. Official Methods of Analysis.

[12] Theophil, E. 2003. Siegfried Hauptmann he Chemistry of Heterocycles' Ed ${ }^{2}$., Germany.

[13] Said, M. B., Mohamed, A. M., Azza, R. M., and Mohamed, H. A. 2016. "Design, Synthesis, Biological Activity and Molecular Modeling of New Heterocyclic Tetrazole Derivatives." Journal of American Science 12 (1): 40-56.

[14] Nisreen, K. A. 2016. "Synthesis and Characterization of Newethyl-2-(5-benzoxazol-2-ylamine-1H-tetrazol-1-yl) Acetate Derivatives." Eng and Tech 34 (2): 287-94.

[15] Carey, B. 2008. "For the Brain, Remembering Is Like Reliving." New York Times.

[16] Ruaa, M. A. 2012. "Synthesis and Characterization of Some Heterocyclic Compounds (Oxazepine, Tetrazole) Derived from Schiff Bases." Journal of Al-Nahrain University 15 (4): 60-7.

[17] Iizuka, M., and Tanaka, H. 1986. Cement admixture. US Patent 4,586,960, filed June 26, 1984, and issued May 6, 1986.

[18] Muzammil, K., Trivedi, P., and Khetani, D. B. 2015. "Synthesis and Characterization of Schiff Base M-nitro Aniline and Their Complexes" Res. J. Chem. Sci. 5 (5): 52-5.

[19] Evanston Public Library Board of Trustees. 2008. "Evanston Public Library Strategic Plan, 2000-2010: A Decade of Outreach.” Evanston Public Library. Accessed July 19, 2008.

[20] Chou, L., McClintock, R., Moretti, F., and Nix, D. H. 1993. "Technology and Education: New Wine in New Bottles: Choosing Pasts and Imagining Educational Futures." Columbia University Institute for Learning Technologies. Accessed

[21] Fine, L. 2005. "Einstein Revisited." J. Chem. Educ. 82: 1601-11. Accessed October 15, 2005.

[22] Young, C. A., and Jordan, T. S. 1995. Cyanide Remediation: Current and Past Technologies. Proceedings of the 10th Annual Conference on Hazardous Waste Research. 